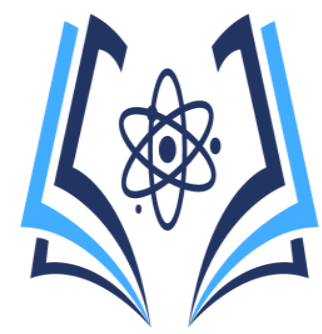

\title{
El síndrome de burnout y sus consecuencias en la salud mental. Una propuesta de operacionalización para su estudio
}

\section{Burnout syndrome and its consequences on mental health. An operationalization proposal for your study}

\section{Síndrome de burnout.}

\author{
Reynier García Rodríguez, Dr. C ${ }^{1}$ \\ Roberth Patricio Zambrano Ubillus, Dr ${ }^{2}$ \\ Karina Yadira Villafuerte García. Lic. ${ }^{3}$ \\ Mauricio Andaluz Granda, Ing. ${ }^{4}$ \\ ${ }^{1}$ Instituto Superior Universitario Portoviejo. reygarod79@gmail.com, ORCID: https://orcid.gob/0000- \\ 0002-4160-5749
}

${ }^{2}$ Instituto Superior Universitario Portoviejo. roberthzamubi@ hotmail.com, ORCID: https://orcid.gob/0000-0002-7526-4938

${ }^{3}$ Unidad Educativa Francisco Pacheco. ru.villafuerte@hotmail.com, ORCID: https://orcid.org/0000-00018224-0080

${ }_{4}^{4}$ Instituto Superior Universitario Portoviejo. mandaluz1987@ hotmail.com, ORCID: 0000-0003-12075600

Correo de contacto: reygarod79@gmail.com

Recibido: 12-08-2020

Aprobado: 05-12-2020

\begin{abstract}
Resumen
La sociedad demanda profesionales preparados, capacitados, poseedores de valores; además de una adecuada salud mental. El Instituto Superior Universitario Portoviejo, propuso un proyecto de investigación de tema: Alternativas de desafíos contra el síndrome Burnout. Una propuesta de mejoramiento para la salud mental y emocional dirigida a los profesionales de diversos ámbitos, con un alcance de dos años, que se cumplen en 2022. La propuesta es innovadora, pertinente, actual y contextualizada, que responde a las necesidades de la provincia y del cantón. El objetivo fue: Proponer un sistema de acciones encaminado a la prevención del síndrome de burnout en el personal de salud, a partir de la operacionalización del síndrome de burnout como variable de estudio. Se aplicó una metodología para un estudio descriptivo, prospectivo y transversal en 200 trabajadores del área de la salud; los que se hallaron sensiblemente afectados: agotamiento físico y mental, falta de motivación absoluta, sensibilidad a la crítica, pérdida de energía, descenso en la productividad laboral y estrés. En la teoría hubo divergencia de las opiniones, considerándose el síndrome de burnout como una patología mental
\end{abstract}

crónica y/o aguda, la investigación permitió valorarla como aguda, en tanto se intervenga en pos de la salud mental. Finalmente, el objetivo fue cumplido, al proyectar acciones en forma de sistema encaminado a la prevención del síndrome de burnout en los trabajadores de la salud. Como conclusión, se arribó a una propuesta innovadora de operacionalización del síndrome de burnout como variable de investigación.

Palabras clave: síndrome de burnout, docentes, salud mental, fatiga, motivación
Abstract
Society demands prepared, trained professionals, possessors of values; in addition to adequate mental health. The Portoviejo Higher University Institute, proposed a research project on the topic: Alternatives of challenges against the Burnout syndrome. A proposal for the improvement of mental and emotional health aimed at professionals from various fields, with a scope of two years, which will be completed in 2022. The proposal is innovative, relevant, current and contextualized, which responds to the 
needs of the province and the canton. The objective was: To propose a system of actions aimed at the prevention of burnout syndrome in health personnel, from the operationalization of the burnout syndrome as a study variable. A methodology was applied for a descriptive, prospective and cross-sectional study in 200 health workers; Those who were significantly affected: physical and mental exhaustion, lack of absolute motivation, sensitivity to criticism, loss of energy, decrease in work productivity and stress. In theory there was divergence of opinions, considering burnout syndrome as a chronic and / or acute mental pathology, the research allowed to value it as acute, as long as it intervenes in pursuit of mental health. Finally, the objective was fulfilled, by projecting actions in the form of a system aimed at the prevention of burnout syndrome in health workers. As a conclusion, an innovative proposal was reached for the operationalization of the burnout syndrome as a research variable.

Keywords: burnout syndrome, teachers, mental health, fatigue, motivation

\section{Introducción}

La Organización Mundial de la Salud (OMS) ha reconocido oficialmente al síndrome de burnout como el síndrome del trabajador quemado (OMS, 2019) y lo caracteriza por un desgaste físico del profesional, independientemente su rama de trabajo.

Es interesante, destacar que el término burnout fue enunciado por vez primera en 1974 por Herbert Freudenberger. Este investigador, aludió que el síndrome es producto del estrés laboral de curso crónico, a su vez debuta en el agotamiento emocional, ausencia de la personalidad, disminución de la autoestima, ineficiencia en la realización de las tareas y dificultades en la comunicación interpersonal.

El síndrome de burnout se refiere de manera coloquial al síndrome del trabajador quemado o desgastado. Lo padecen las personas que responden a un estrés prolongado, donde el organismo reacciona antes los factores generadores de estrés, entre ellos: fatiga, ineficacia y hasta la negación de los hechos que lo motivan.

Este síndrome es objeto de estudio en varios países y se ajusta a la clasificación de los problemas relacionados con los afrontamientos a las dificultades de la vida. En Europa se le diagnostica como el síndrome de neurastenia pero con la condición de que su sintomatología se asocie al trabajo (Murga \& Lafuente, 2019), quienes lo catalogan como enfermedad mental.
En el mundo se ha evidenciado el síndrome de burnout, Ecuador no es excepción y las principales problemáticas de vislumbran en las afectaciones en la salud mental y es la antesala de otras patologías a nivel psíquico como insuficiencias en el autocontrol y carencias en el trabajo preventivo relacionado con este síndrome (Méndez, 2019).

Por su parte, Rodríguez, Guevara \& Viramontes (2017) destacaron que en los docentes se revela con mayor nitidez, los signos y síntomas relacionados con el síndrome. A tenor, el trabajo se vuelve agotador, estresante y frustrante, el distanciamiento social, somnolencia y pereza laboral. $\mathrm{Ha}$ resultado interesante, la manifestación de cefalea intensa, trastornos digestivos, falta de energía, problemas de comunicación familiar, dificultad en la concentración y carente creatividad.

Estas dificultades son apreciables en cualquier tipo de educación incluyendo la educación superior. De donde se hace el siguiente análisis, la importancia de la educación en Educador es esencial sin embrago, los docentes son susceptibles al estrés laboral y por ende pueden debutar con el síndrome de burnout, así como su incidencia en la salud mental.

Desde esta lógica se define como problema a investigar: ¿Cómo contribuir a la prevención del síndrome de burnout en trabajadores del área de la salud, a partir de una adecuada operacionalización de dicho síndrome como variable de estudio? que tiene solución al identificar como objeto de estudio: el síndrome de burnout, campo de investigación: la salud mental del personal de salud.

La presente investigación tiene como objetivo: Proponer un sistema de acciones encaminado a la prevención del síndrome de burnout en el personal de salud, a partir de la operacionalización del síndrome de burnout como variable de estudio.

\section{Materiales y métodos}

Se realizó un estudio descriptivo, prospectivo y transversal en 200 personas del área de salud. En el orden de los métodos teóricos se emplearon: análisis - síntesis, inductivo - deductivo e histórico - lógico para el alcance de la descripción del objeto y campo de investigación y finalmente arribar a conclusiones. En el orden de los métodos empíricos, se empleó una encuesta con la intención de discernir cuáles son las generalidades y particularidades de los docentes que participaron en la investigación.

La investigación se ha proyectado desde inicios de 2020 hasta 2022, tomándose como referencia 
instituciones del área de salud fiscales y privadas. Los criterios de inclusión: género, edad, carga laboral, descanso activo, ambiente laboral y recursos personológicos.

Se procedió al diseño de una base de datos para realizar el análisis estadístico, se utilizó el programa Microsoft Office Excel. Se estimaron los porcentajes como medida de resumen de la estadística descriptiva y se estimaron las frecuencias relativas. No se tuvieron en cuenta estadígrafos pues responde a dos variables cualitativas. Se emplearon principios éticos: confiabilidad de los criterios obtenidos y consentimiento informado.

\section{Resultados}

La aplicación de la encuesta arrojó resultados interesantes que giran en torno al conocimiento sobre el síndrome de Burnout, donde 150 personas no conocen el tema $(75 \%)$ y 50 si conocen sobre el síndrome (25\%). Resultó interesante que el $25 \%$ solo ha escuchado el nombre pero no lo asocian a un síndrome del que todos pueden ser susceptibles, conllevándolos a enfermedades mentales a partir de un estrés agudo y persistente.

En este orden de análisis, se determinó que los síntomas más frecuentes durante la pandemia fueron: agotamiento físico y mental (100\%), falta de motivación absoluta (30\%), sensibilidad a la crítica (20\%), pérdida de energía (85\%), descenso en la productividad laboral $(90 \%)$ y estrés (100\%). Vale destacar, que el 100\% de los encuestados revelaron al menos un síntoma y es interesante notar que todos declararon que presentaron agotamiento físico y mental, acompañado del estrés de manera permanente.

Al no existir claridad en el conocimiento del síndrome de Burnout, de manera empírica identifican algunos síntomas relacionados con la creencia que tienen sobre el síndrome, sin llegar a aseveraciones reales o definitivas. En consecuencia, el $95 \%$ destacó el agotamiento físico y mental, así como los dolores de cabeza; como principales síntomas y el 5\% refirió que los mareos son frecuentes dada la cantidad de información que deben analizar para realizar un trabajo o sencillamente al recibir un sinnúmero de orientaciones que se contraponen entre ellas. Vale señalar que el $100 \%$ de los encuestados ponderan al estrés como una sintomatología general, que entorpece su desempeño profesional.

Por consenso, declararon que en tiempos de pandemia y distanciamiento social, se trabaja el triple de lo que se trabajaba en otras realidades, se ven afectados por las orientaciones que reciben de diversas fuentes. Esta situación devela, a juicio del 100\%, que el síndrome de burnout puede aparecer por el estrés recurrente y la presión de permanecer varias horas de trabajo nocturno (velas).

El $95 \%$ de los encuestados reveló que desconectarse de sus actividades no previene la aparición de este síndrome, por el contrario. El no cumplir con sus obligaciones como servidores públicos puede interferir en su estabilidad laboral. Sin dudas, el 100\% consideró que la presión acompañada de estrés, debuta en afectaciones agudas y/o crónicas en la salud mental y emocional.

El $80 \%$ refirió que el síndrome de burnout se puede dividir en dos tipos, poniendo énfasis en el de mayor actividad, pero no supieron explicar que este se debe a los factores que rodean al ejercicio de la profesión. En cambio, el 100\% refirió que el síndrome más predominante y fuerte es el relacionado con los factores sociales y psicológicos, con sentimientos de negación, decepción, apatía, comunicación poco asertiva y disociación en el carácter y otros recursos de la personalidad.

Las recomendaciones estuvieron dirigidas al mejoramiento en el ámbito social y docente.

Ampliar el contacto social, ser un buen oyente de noticias atrayentemente positivas.

Evitar personas que distraigan o juzguen por la situación en que se pueda encontrar.

Expandir la confianza con los amigos, fortaleciendo las relaciones interpersonales.

Organizar el trabajo, pidiendo ayuda a través de la sana amistad con las que laboramos.

Se recomienda: ampliar la red social, nunca permanecer solos, ejercitarse físicamente, reorganizar las prioridades y metas, autovalorar las funciones, ejercitar los talentos propios.

Se trata de desarrollar en el individuo conductas que eliminen las fuentes de estrés. El utilizar estrategias de afrontamiento adecuadas ayuda a prevenir el desarrollo del burnout, siendo también necesario entrenamientos en cuanto a la solución de problemas, el adecuado manejo del tiempo y el asertividad.

Esta intervención se recomienda utilizarla en cualquier momento del desarrollo del síndrome, incluso antes de que se muestren los primeros síntomas del Burnout, pues al conseguir estrategias apropiadas para manejarlo se podrá prevenir su aparición.

Es importante, ser asertivo: es importante adoptar un estilo asertivo ante nuestros compañeros y superiores con el fin de que las relaciones laborales y el trabajo en sí no se vea deteriorado por los malos entendidos. Poner límites: también es fundamental no comprometernos a hacer cosas sobre las que realmente no nos sentimos 
capacitados o bien no consideramos que sea nuestro trabajo hacerlo.

Ajustar las expectativas: en lo que respecta a las expectativas e ideales estas no deben suprimirse, pero debemos saber ajustarlas. Es bueno que tengamos una meta a la que queramos llegar, un ideal de trabajo, pero también es importante plantearse si lo que tenemos no está mal. La expectativa laboral nos debe ayudar a crecer, no a decrecer profesionalmente.

Análisis y discusión

Los estudios de Cialzeta (2013) dirigieron la atención al síndrome de Burnout en médicos mexicanos. Este autor señaló que el síndrome estudiado es una respuesta inadecuada al estrés emocional crónico consecuencia de elevados niveles de tensión en el trabajo, frustración personal y actitudes inadecuadas de enfrentamiento a situaciones conflictivas.

Hasta este orden de análisis se coincide, pues uno de los tipos de estrés, de curso crónico, pues la tensión es fuerte y prolongable puesto que se desconoce cuándo se regresará a la nueva normalidad.

Estudios recientes en México y Ecuador, revelaron que el síndrome de burnout es un fenómeno relacionado con el estrés laboral (Rodríguez, Guevara \& Viramontes, 2017). Los investigadores aluden a que es una manifestación aguda, al referirse a los médicos, añaden que, tal vez en otras esferas laborales puede ser crónico en dependencia del entorno donde se desempeña profesionalmente. Elementos claves para la generación de estrés según la investigación son: el exceso de pacientes con la Covid.

Los resultados encontrados a través del presente estudio, solo se coincide con las largas jornadas laborales. Las decisiones administrativas, en ocasiones arbitrarias, que entorpecen los procedimientos laborales.

A la luz de ambos criterios, surge una interrogante: ¿el síndrome de burnout crónico podrá evidenciarse en los médicos? Sí, cuando hay sensación de estar fuera de lugar, emociones soslayadas, impotencia, desesperanza, desmotivación, depresión, daño emocional y psicológico (Guerri, 2020). Se llega a una conclusión parcial que alude a tomar en cuenta al hombre como ser social y brindarle una atención personalizada y humanizada que coadyuve a la prevención del síndrome de burnout.

Se comparte una propuesta de operacionalización de la variable: Síndrome de burnout (Tabla 1)

Tabla 1. La operacionalización de la variable: Síndrome de burnout

\begin{tabular}{|c|c|c|c|c|}
\hline Variable & Definición & Dimensiones & Indicadores & Escala \\
\hline \multirow{7}{*}{$\begin{array}{l}\text { Síndrome de } \\
\text { burnout }\end{array}$} & \multirow{7}{*}{$\begin{array}{c}\text { "Es una respuesta } \\
\text { caracterizada por } \\
\text { agotamiento emocional, } \\
\text { despersonalización y baja } \\
\text { realización personal en el } \\
\text { trabajo, que puede ocurrir } \\
\text { entre el personal de salud, } \\
\text { debido a las tareas diarias } \\
\text { circunscritas al servicio de } \\
\text { personas" (Olivares, 2017) }\end{array}$} & Agotamiento emocional & Recursos emocionales & Nominal \\
\hline & & & $\begin{array}{c}\text { Fatiga } \\
\text { Esfuerzo emocional }\end{array}$ & Nominal \\
\hline & & Despersonalización & $\begin{array}{l}\text { Conducta } \\
\text { Sensaciones }\end{array}$ & Nominal \\
\hline & & & Actitudes & Nominal \\
\hline & & Realización personal & Vivencias afectivas & Nominal \\
\hline & & & Autoestima & Nominal \\
\hline & & & Motivación & Nominal \\
\hline
\end{tabular}

Fuente: Elaboración propia 
Los estudios de Menghi \& Oros (2019) apuntaron a los sinónimos relacionados con el síndrome de burnout, de este modo aseguraron que es un desgate físico y mental, es consumirse sobrepasando los niveles de estrés admisibles por los seres humanos, en algunos casos irreversibles

Desde el siglo XX a la actualidad se ha estudiado en dimensiones de interés notorio como son: el cansancio emocional que se revela por las exigencias de trabajo y la presión que se ejerza sobre la persona, la despersonalización que valora la indiferencia y la apatía social y la insuficiente realización como persona, que tiene en cuenta la baja autoestima que impiden visionar el éxito y la realización personal (Romero et. al., 2019).

¿Qué se requiere entonces para contrarrestar los efectos del estrés que puede a corto plazo convertirse en el síndrome de burnout?

Desarrollar en los docentes la capacidad resiliente, al mencionar la resiliencia es significar que es la capacidad que puede desarrollar el docente para enfrentar situaciones difíciles y retadoras, y así superar los obstáculos de la vida (Gambarte, 2019).

A través del estudio se han vislumbrado características de la resiliencia, que implica:

El logro de una perspectiva dinámica, interactiva y de desarrollo humano.

La conquista permanente de la salud mental y la adaptación social.

El abrir ventanas al optimismo, a la creatividad y a autoconfianza.

El mejoramiento constante como ser humano y profesional.

En este orden de análisis, la resiliencia no es estática, es susceptible de estudio y aplicación permanente, hay que repensarla porque va desde las capacidades a las competencias, forma parte de las competencias de vida, es impostergable e implica ir hacia adelante, reconquistarse, reinventarse, ser mejor como persona en el diario vivir.

El síndrome de burnout precisa de la prevención consciente

El mejoramiento profesional y humano de las personas.

La renuncia a la cultura de la queja.

El logro de aprendizajes permanentes y enriquecidos.

La concreción del trabajo en contexto, personalizado, tecnológico.

La búsqueda de soluciones urgentes.
El pensamiento de diseño: empatizar, definir, idear, prototipar, evaluar.

La armonía colectiva, la sana convivencia y las relaciones fraternas.

Se propone un sistema de acciones relacionado con:

Desarrollo de capacitaciones dirigidas y personalizadas.

Consultorías pedagógicas y psicológicas dirigidas.

Trabajo psicológico, en función de la resiliencia. Hasta este orden de análisis se discurre en la salud mental, que a la luz de los aportes de Marenco-Escuderos \& Ávila-Toscano (2016), incluye el bienestar emocional, psicológico y social; donde es esencial la manera en que se piensa, se siente y se actúa cuando se asumen los retos de la vida. En este sentido, implica el manejo de estrés y la toma de decisiones, puesto que la salud mental es importante para cada etapa de la vida.

\section{Conclusiones}

- La totalidad de las personas se encontraron sensiblemente afectados, en este sentido los principales síntomas identificados fueron: agotamiento físico y mental, falta de motivación absoluta, sensibilidad a la crítica, pérdida de energía, descenso en la productividad laboral y estrés $(100 \%)$.

- Resultó interesante cada posición epistemológica analizada, las que se discutieron oportunamente por la divergencia de las opiniones, considerándose el síndrome de burnout como una patología mental crónica y/o aguda, la investigación permitió valorarla como aguda, en tanto se intervenga en pos de la salud mental. Si no se le presta atención, puede desencadenar en una enfermedad psiquiátrica.

- La aplicación de la encuesta como método empírico arrojó información de alta valía dirigida a una propuesta operativa de sistema de acciones encaminado a la prevención del síndrome de burnout del personal de salud implicado, con énfasis en los médicos. 


\section{Referencias bibliográficas}

- Acosta-Fernández, M., Parra-Osorio, L., Restrepo-García, J. I., Pozos-Radillo, B. E., de los Ángeles Aguilera-Velasco, M., \& Torres-López, T. M. (2017). Condiciones psicosociales, violencia y salud mental en docentes de medicina y enfermería. Salud Uninorte, 33(3), 344-354.

- Gambarte, M. I. G. (2019). Liderazgo Pedagógico en Educación Secundaria: Aportaciones desde la Evaluación de Burnout-Resiliencia en Docentes. Management, 7, 104-134.

- Guevara-Manrique, A. C., Sánchez-Lozano, C. M., \& Parra, L. (2014). Estrés laboral y salud mental en docentes de primaria y secundaria. Revista colombiana de salud ocupacional, 4(4), 3032.

- Marenco-Escuderos, A. D., \& Ávila-Toscano, J. H. (2016). Burnout y problemas de salud mental en docentes: diferencias según características demográficas y sociolaborales. Psychologia. Avances de la disciplina, 10(1), 91-100.

- Méndez Venegas, J. (2019). Estrés laboral o síndrome de burnout.

- Menghi, M. S., \& Oros, L. B. B. (2019). Satisfacción laboral y síndrome de burnout en docentes de nivel primario. Revista de Psicología, 10(20), 47-59.

- Morales Ortiz, J., Muñoz Martínez, S., \& Pérez Sánchez, S. (2020). Abre tu mente en modo positivo. Promoción de salud mental en jóvenes: guía para docentes y familias.

- Murga, Í., \& Lafuente, J. V. (2019). De la neurastenia a la enfermedad postesfuerzo: evolución de los criterios diagnósticos del síndrome de fatiga crónica/encefalomielitis miálgica. Atención Primaria, 51(9), 579-585.

- Rodríguez Ramírez, J. A., Guevara Araiza, A., \& Viramontes Anaya, E. (2017). Síndrome de burnout en docentes. IE Revista de Investigación Educativa de la REDIECH, 8(14), 45-67.

- Romero Fernández, A., Pimienta Concepción, I., Ramos Argilagos, M., Sánchez Garrido, A., \& González Salas, R. (2019). Niveles de síndrome de burnout en docentes de una universidad ecuatoriana. Dilemas

- Contemporáneos: Educación, Política y Valores, 6(3). 